\title{
Impact of smoking in patients undergoing transcatheter aortic valve replacement
}

\author{
Manyoo Agarwal ${ }^{1}$, Sahil Agrawal ${ }^{2}$, Lohit Garg ${ }^{3}$, Guy L. Reed ${ }^{4}$, Rami N. Khouzam ${ }^{4}$, Uzoma N. Ibebuogu ${ }^{4}$ \\ ${ }^{1}$ Department of Internal Medicine, University of Tennessee Health Science Center, Memphis, TN, USA; ${ }^{2}$ Division of Cardiovascular Medicine, \\ Department of Internal Medicine, St Luke's University Health Network, Bethlehem, PA, USA; ${ }^{3}$ Division of Cardiovascular Medicine, Department \\ of Internal Medicine, Lehigh Valley Health Network, Allentown, PA, USA; ${ }^{4}$ Department of Internal Medicine, Division of Cardiovascular Diseases, \\ University of Tennessee Health Science Center, Memphis, TN, USA \\ Contributions: (I) Conception and design: All authors; (II) Administrative support: All authors; (III) Provision of study materials or patients: All \\ authors; (IV) Collection and assembly of data: All authors; (V) Data analysis and interpretation: All authors; (VI) Manuscript writing: All authors; (VII) \\ Final approval of manuscript: All authors. \\ Correspondence to: Uzoma N. Ibebuogu, MD, FACC, FSCAI. Division of Cardiovascular Diseases, Department of Internal Medicine, University of \\ Tennessee Health Science Center, 956 Court Avenue, Suite D 334, Memphis, TN 38163, USA. Email: ibebuogu@gmail.com or uibebuog@uthsc.edu.
}

\begin{abstract}
Background: The paradox that smokers have better clinical outcomes in cardiovascular diseases remains controversial. No literature exists studying impact of smoking on outcomes following transcatheter aortic valve replacement (TAVR).

Methods: We performed an electronic search of the 2011-2012 National Inpatient Sample (NIS) database to identify all TAVR hospitalizations. Outcomes were measured comparing smokers to non-smokers.

Results: A total of 8,345 TAVR hospitalizations were identified with $24 \%$ being smokers. Compared to non-smokers, smokers were younger $(80.4 \pm 8.8$ vs. $81.4 \pm 9.2$ years, $\mathrm{P}<0.001)$, were more often men $(63.6 \%$ vs. $47.8 \%, \mathrm{P}<0.001)$, and had a higher disease burden. Despite a higher disease burden, smokers had lower post procedure stroke $(2.8 \%$ vs. $3.1 \%)$, hemorrhage events $(28.2 \%$ vs. $32.0 \%, \mathrm{P}<0.05)$ and lower all cause inhospital mortality $(1.2 \%$ vs. $5.7 \%$, adjusted odds ratio $0.21,95 \%$ CI: $0.13-0.32, \mathrm{P}<0.001)$ compared to nonsmokers.
\end{abstract}

Conclusions: Despite having a higher cardiovascular disease burden, smokers had better outcomes compared to non-smokers. Therefore the smoker's paradox is applicable in the TAVR cohort.

Keywords: Smoker's paradox; transcatheter aortic valve replacement (TAVR); aortic stenosis; National Inpatient Sample database

Submitted Jun 30, 2017. Accepted for publication Nov 10, 2017.

doi: $10.21037 /$ atm.2017.11.32

View this article at: http://dx.doi.org/10.21037/atm.2017.11.32

\section{Introduction}

Transcatheter aortic valve replacement (TAVR) has become a standard of care for severe aortic stenosis patients, with intermediate, high or prohibitive risk for surgical aortic valve replacement (1). Smoking is an established risk factor for the development of cardiovascular diseases and has been shown to accelerate the progression of aortic stenosis (2). Interestingly, data exists supporting better survival outcomes in smokers compared to no-smokers with cardiovascular diseases and after cardiovascular interventions $(3,4)$. This thought-provoking observation has been termed as the "Smoker's paradox." While smoking related diseases such as chronic obstructive pulmonary disease has been associated with adverse clinical outcomes in patients undergoing TAVR, to the best of our knowledge there is no existing literature on the impact of smoking on in-hospital mortality in patients undergoing TAVR. Therefore, we analyzed the Nationwide Inpatient Sample (NIS) database from 2011-2012 to determine the association of smoking status 
with in-hospital clinical outcomes in TAVR patients.

\section{Methods}

All TAVR admissions between January 1, 2011 and December 31, 2012 were identified in the NIS database using the International Classification of Diseases, ninth edition, and Clinical Modification (ICD-9-CM) codes $(35.05,35.06)$. The study protocol was exempted from review by the Institutional Review Board of the University of Tennessee Health Science Center, Memphis. Baseline patient demographic characteristics (age, sex, and race), and comorbidities (as listed in Table 1) were extracted from the NIS database. Our outcome measures included allcause in-hospital mortality, defined as "died" during the index hospitalization in the database, acute cerebrovascular accidents (CVAs) (CCS-109) and post-procedure hemorrhage (identified using ICD-9 CM codes-998.11, 998.12, 285.1, 568.81). Baseline patient demographics and comorbidities were compared using Pearson's Chisquare test for categorical variables and Student's t-test for continuous variables. In addition to patient demographics and comorbidities, smoking was used as an independent variable in a multivariable unconditional regression model to examine predictors of primary and secondary outcomes. Statistical analysis was performed using SPSS 23.0 (IBM corp., Armonk, NY, USA). We used a 2-sided P value of $<0.05$ to identify statistical significance.

\section{Results}

Among the 8,345 patients who underwent the TAVR procedure, $24 \%$ were smokers $(n=2,004)$ and $76 \%$ were non-smokers $(\mathrm{n}=6,341)$. The mean age of the overall study population was $81.1 \pm 9.1$ years, with $51.6 \%$ being male and $83.6 \%$ being Caucasian. Patient demographics and comorbidities are listed in Table 1. Smokers were more likely to be male, younger and had higher prevalence of hypertension, dyslipidemia, obesity, coronary artery disease, peripheral arterial disease and chronic pulmonary disease than non-smokers. The prevalence of hypertension, hyperlipidemia, obesity, peripheral arterial disease, carotid artery disease, coronary artery disease, cerebrovascular disease and chronic obstructive pulmonary disease, was higher in smokers than non-smokers (all $\mathrm{P}<0.001$ ). Smokers were less likely to have congestive heart failure and renal failure (all $\mathrm{P}<0.001$ ). Despite having a higher prior medical history of CVA, smokers compared to non-smokers were less likely to have acute CVA $(2.8 \%$ vs. $3.1 \%)$, post procedure hemorrhage ( $28.2 \%$ vs. $32.0 \%)$ or in-hospital death $(1.2 \%$ vs. $5.7 \%$, adjusted OR $0.21,95 \%$ CI, $0.13-0.32)$ (Table 2).

\section{Discussion}

We observed that smokers had lower in-hospital mortality and adverse clinical outcomes compared to non-smokers. This observation was valid even after multivariate adjustment for patient demographics and comorbidities. Hence, we concluded that smoker's paradox does exist in TAVR patients.

Currently there is no data on the interaction between smoking and TAVR. In patients undergoing surgical aortic valve replacement, Saxena et al., reported no worse perioperative clinical outcomes in smokers when compared to non-smokers in a study based on Australian and New Zealand Society of Cardiac and Thoracic Surgeons (SCTS) Cardiac Surgery Database Program (5). Similarly, another single center study reported no effect of smoking status on post-operative risk in patients undergoing valve (6). Other studies analyzing the impact of smoking on survival after cardiovascular events such as myocardial infarction, stroke, percutaneous coronary intervention and thrombolysis have previously reported findings supporting the smoker's paradox $(3,4)$. Generally, better survival in smokers has been related to factors such as younger age and lesser comorbidities (3). Nevertheless, we found a strong residual survival benefit in smokers even after multivariate adjustment for multiple factors including age and comorbidities. Hence, lower mortality in smokers in our study may be due to unmeasured confounding, or possibly a protective association.

It is critically important to emphasize that this paradoxical association of smoking with favorable survival in TAVR should not be seen as encouragement for smoking. These results suggest a strong association between smoking and in-hospital adverse clinical outcomes in TAVR, but these results do not assume causation. Also, extensive literature supports that smoking is an established risk factor for the epidemiology of $\mathrm{CV}$ diseases, as well as its morbidity and mortality. However the existence of smoker's paradox deserves attention and needs further research to better explain the mechanism(s) behind this interesting observation. 
Table 1 Comparison of TAVR patient's demographics and comorbidities based on smoking status

\begin{tabular}{|c|c|c|c|c|}
\hline Characteristic & Overall $(n=8,345)$ & Smokers $(n=2,004)$ & Non-smokers $(n=6,341)$ & $\mathrm{P}_{\text {value }}^{+}$ \\
\hline Female (\%) & 48.4 & 36.4 & 52.2 & $<0.001$ \\
\hline Race/ethnicity (\%) & & & & $<0.001$ \\
\hline White & 83.6 & 82.7 & 83.9 & \\
\hline Hispanic & 4.3 & 3.0 & 4.8 & \\
\hline Other & 8.4 & 11.8 & 7.2 & \\
\hline \multicolumn{5}{|l|}{ Comorbidities* (\%) } \\
\hline Hypertension & 79.8 & 84.7 & 78.2 & $<0.001$ \\
\hline Obesity & 12.7 & 15.9 & 11.7 & $<0.001$ \\
\hline Congestive heart failure & 1.5 & 0.5 & 1.9 & $<0.001$ \\
\hline Atrial fibrillation & 44.5 & 43.9 & 44.7 & 0.52 \\
\hline Peripheral arterial disease & 29.6 & 32.6 & 28.7 & 0.001 \\
\hline Carotid artery disease & 5.9 & 8.4 & 5.1 & $<0.001$ \\
\hline Chronic pulmonary disease & 34.4 & 48.1 & 30.0 & $<0.001$ \\
\hline Cerebrovascular disease history & 12.2 & 13.7 & 11.8 & 0.02 \\
\hline Renal failure & 36.3 & 32.5 & 37.5 & $<0.001$ \\
\hline \multicolumn{5}{|l|}{ TAVR approach (\%) } \\
\hline Transfemoral & 83.2 & 81.4 & 83.8 & 0.011 \\
\hline Transapical & 17.0 & 19.1 & 16.3 & 0.004 \\
\hline
\end{tabular}

*, co-morbidities were extracted from the database using International Classification of Diseases, Ninth Edition, Clinical Modification Diagnosis or Clinical Classification Software codes; ', P value representing difference between smokers and non-smokers. MI, myocardial infarction; CABG, coronary artery bypass grafting; PCI, percutaneous coronary intervention; SD, standard deviation; TAVR, transcatheter aortic valve implantation. 
Table 2 In-hospital outcomes in non-smokers and smokers undergoing transcatheter aortic valve replacement

\begin{tabular}{lccccc}
\hline Outcome & $\begin{array}{c}\text { Smokers } \\
(\mathrm{n}=2,004)(\%)\end{array}$ & $\begin{array}{c}\text { Non-smokers } \\
(\mathrm{n}=6,341)(\%)\end{array}$ & \multicolumn{2}{c}{ Odds ratio $(95 \% \mathrm{Cl})$} & Adjusted $^{*}$ \\
\hline In-hospital mortality & 1.2 & 5.7 & & $0.21(0.14-0.32)$ & $0.21(0.13-0.32)$ \\
Post-procedure hemorrhage & 28.2 & 32.0 & $0.83(0.75-0.93)$ & $0.88(0.78-0.98)$ & $<0.001$ \\
Acute cerebrovascular events & 2.8 & 3.1 & $0.79(0.58-1.09)$ & $0.96(0.69-1.34)$ & 0.81 \\
\hline
\end{tabular}

*, adjusted for patient demographics, comorbidities and TAVR approach. Cl, confidence interval; TAVI, transcatheter aortic valve implantation.

\section{Study limitations}

The main strength of our study is a large nationalized unselected sample size. However, lack of clinical information because of the administrative nature of the database along with the possibility of coding errors should be noted. In addition, we did not have any information regarding smoking status (former or current), number of pack years, and medications patient were using at the time of admission, procedure, and discharge. Finally, NIS is limited to in-hospital events and consequently, no information relating to long-term effects is available.

\section{Conclusions}

In our large nationalized observational study, smoking was independently associated with better clinical outcomes, supporting the smoker's paradox in patients undergoing TAVR.

\section{Acknowledgements}

None.

\section{Footnote}

Conflicts of Interest: The authors have no conflicts of interest to declare.

Ethical Statement: The study protocol was exempted from

Cite this article as: Agarwal M, Agrawal S, Garg L, Reed GL, Khouzam RN, Ibebuogu UN. Impact of smoking in patients undergoing transcatheter aortic valve replacement. Ann Transl Med 2018;6(1):2. doi: 10.21037/atm.2017.11.32 review by the Institutional Review Board of the University of Tennessee Health Science Center, Memphis.

\section{References}

1. Nishimura RA, Otto CM, Bonow RO, et al. 2014 AHA/ ACC Guideline for the Management of Patients With Valvular Heart Disease: executive summary: a report of the American College of Cardiology/American Heart Association Task Force on Practice Guidelines. Circulation 2014;129:2440-92.

2. Palta S, Pai AM, Gill KS, et al. New insights into the progression of aortic stenosis: implications for secondary prevention. Circulation 2000;101:2497-502.

3. Gupta T, Kolte D, Khera S, et al. Smoker's Paradox in Patients With ST-Segment Elevation Myocardial Infarction Undergoing Primary Percutaneous Coronary Intervention. J Am Heart Assoc 2016;5. pii: e003370.

4. Kufner A, Nolte CH, Galinovic I, et al. Smokingthrombolysis paradox: recanalization and reperfusion rates after intravenous tissue plasminogen activator in smokers with ischemic stroke. Stroke 2013;44:407-13.

5. Saxena A, Shan L, Dinh DT, et al. Impact of smoking status on early and late outcomes after isolated aortic valve replacement surgery. J Heart Valve Dis 2013;22:184-91.

6. Al-Sarraf N, Thalib L, Hughes A, et al. Lack of correlation between smoking status and early postoperative outcome following valve surgery. Thorac Cardiovasc Surg 2008;56:449-55. 